TITLE:

\title{
Flux pinning in Nb-Ti based nanoscale superconducting multilayers
}

\section{AUTHOR(S):}

Oguma, H; Osamura, K; Sakai, A; Otsuka, H; Ito, I

\section{CITATION:}

Oguma, $\mathrm{H}$...[et al]. Flux pinning in Nb-Ti based nanoscale superconducting multilayers. IEEE TRANSACTIONS ON APPLIED SUPERCONDUCTIVITY 2001, 11(1): 3804-3807

\section{ISSUE DATE:}

2001-03

URL:

http://hdl.handle.net/2433/39944

\section{RIGHT:}

(c)2001 IEEE. Personal use of this material is permitted. However, permission to reprint/republish this material for advertising or promotional purposes or for creating new collective works for resale or redistribution to servers or lists, or to reuse any copyrighted component of this work in other works must be obtained from the IEEE. 


\title{
Flux Pinning in Nb-Ti based Nanoscale Superconducting Multilayers
}

\author{
Hidetaka Oguma, Kozo Osamura, Akira Sakai, Hiroaki Otsuka and Ikuo Ito
}

\begin{abstract}
Nanoscale $\mathrm{Nb}-\mathrm{Ti} / \mathrm{Nb} / \mathrm{Cu}-\mathrm{Ni}-\mathrm{Si}-\mathrm{Zn}$ multilayers have been fabricated by means of a plastic deformation technique where a precipitation - hardening copper alloy was used. When the thickness dependence of the global pinning force was examined at constant magnetic fields, it increased monotonically with increasing $\mathrm{Nb}-\mathrm{Ti}$ layer thickness at $5 \mathrm{~T}$ from 4.8 to $120 \mathrm{~nm}$, but it showed a maximum at $24 \mathrm{~nm}$ at $1.5 \mathrm{~T}$. The critical temperature $\left(T_{s}\right)$ decreased with decreasing thickness when the layer thickness was smaller than $20 \mathrm{~nm}$. The present experimental results suggested that the major flux pinning is attributed to the superconducting/normal interface and the proximity effect resulted in the degradation of $T_{k}$.
\end{abstract}

Index Terms- artificial pinning, Nb-Ti alloy, nano-scale multilayer, proximity effect

\section{INTRODUCTION}

A high performance magnetic shield composed of superconducting materials is used for the magnetic shielding at high magnetic fields and for the severe magnetic shielding. The principle is explained as follows. The magnetic flux density becomes zero at the distance from the surface $d_{\mathrm{p}}=B /\left(\mu_{\mathrm{o}} J_{\mathrm{c}}\right)$ when the external magnetic field $(B)$ penetrated in the superconducting sheet. Accordingly, the use of thinner shielding sheet is possible when the sheet has higher critical current density $\left(J_{c}\right)$. Also the good mechanical deformability is requested for fabricating a complicated shielding container, for instance. At present, $\mathrm{Nb}-\mathrm{Ti}$ composite superconducting sheets are the best materials for magnetic shielding.

The commercial $\mathrm{Nb}-\mathrm{Ti}$ sheets have fairly high critical current density [1], where the ribbon - like Ti precipitates act effectively as magnetic flux pinning centers. Due to the precipitation treatment, the maximum volume fraction of $\mathrm{Ti}$ precipitates is only limited up to $25 \%$ [2]. In order to realize higher critical current density, an increase of number density of pinning sites is highly desired. One of several relevant ideas is to create artificial pinning centers ( APC ). The thin $\mathrm{Nb}$ filaments are embedded regularly and densely in the $\mathrm{Nb}-\mathrm{Ti}$ matrix along the wire[3]. Those $\mathrm{Nb}$ filaments behave like dots

Manuscript received Sept. 18, 2000

H.Oguma and K.Osamura are with Department of Materials Science and Engineering, Kyoto University, Kyoto 606-8501, Japan (e-mail: osamura@hightc.mtl.kyoto-u.ac.jp)

A.Sakai is with Mesoscopic Materials Research Center, Faculty of Engineering, Kyoto University, Kyoto 606-8501, Japan (e-mail: sakai@mesostm.mtl.kyto-u.ac.jp)

H.Ohtsuka and I.Itoh are with Advanced Technology Research Laboratories, Nippon Steel Corp., Futtsu, Chiba 293-8511, Japan (e-mail: hotsuka@re.nsc.co.jp) in the cross section perpendicular to the wire axis. From their geometrical arrangement, they are called island type APC. The other is called lamella type APC. Two $\mathrm{Nb}-\mathrm{Ti}$ and $\mathrm{Nb}$ sheets are stacked mutually, and then mechanically pressed and cut to get strips. Their strips are composed to the round wires[4]. Till now, many efforts have been concentrated to fabricate the wires with APC. Our research subject, however, is to improve the superconducting sheets. The simple idea is to design the multilayer of superconducting (SC)/normal (N) sheets, by which the volume fraction of superconductor can reach to $50 \%$. The optimum pinning force, however, can be theoretically explained in terms of the ratio of coherence length and the ratio of layer thickness between the SC and the $\mathrm{N}$ layers. The quantitative calculation has been performed elsewhere [5]. Further it is easy to control the period and structure of multilayers in order to realize the best shielding performance for a given magnetic field.

In the present study, we fabricated the nanoscale multilayer sheets by means of the thermomechanical technique and investigated their superconducting properties as well as microstructure.

\section{EXPERIMENTAL PROCEDURE}

The $\mathrm{Nb}-\mathrm{Ti}\left(\mathrm{d}_{\mathrm{s}}\right) / \mathrm{Nb}\left(\mathrm{d}_{\mathrm{b}}\right) / \mathrm{Cu}$-alloy $\left(\mathrm{d}_{\mathrm{n}}\right)$ multilayers were fabricated by using the thermomechanical treatment technique, where $d_{s}, d_{b}$ and $d_{n}$ are the layer thickness and the ratio $d_{s} / d_{b} / d_{n}$ was kept constant as 10/1/10. As shown in Fig. 1, a fundamental assembly consisted of $\mathrm{Nb}-46.5 \% \mathrm{Ti}(0.8 \mathrm{~mm}) /$ $\mathrm{Nb}(0.05 \mathrm{~mm}) / \mathrm{Cu}$ alloy $(0.8 \mathrm{~mm}) / \mathrm{Nb}(0.05 \mathrm{~mm})$ unit, where the original thickness of each sheet is indicated in the parentheses. A Cu sheet $(8 \mathrm{~mm})$ was inserted after every 30 stacks of the above unit. The thickness of the total assembly was $66.2 \mathrm{~mm}$. Here the chemical composition of $\mathrm{Cu}$ alloy was $\mathrm{Cu}-3 \% \mathrm{Ni}-0.6 \% \mathrm{Si}-0.2 \% \mathrm{Zn}$ ( in mass \%), which has been the best selection for obtaining nanoscale homogeneous multilayer. The present alloy is known as Corson alloy used to the age-hardenable high couductivity metal. After casing them in the $\mathrm{Cu}$ container and pressing at room temperature, the composite was hot-rolled at $1073 \mathrm{~K}$ to $34 \mathrm{~mm}$ thickness sheet and then cold-rolled to $0.2 \mathrm{~mm}$ thickness. Following the cold-rolling process, several samples were annealed at $773 \mathrm{~K}$ for $7.2 \mathrm{ks}$. These are designated annealed samples hereafter in the present paper. The other samples were not annealed. Then all the thin samples were cut into small square plates with dimensions of $15 \mathrm{~mm} \times 20 \mathrm{~mm}$. The thin plates were stacked 20 times and cased in the $\mathrm{Cu}$ container. This assembly was hot-pressed at $823 \mathrm{~K}$ for $7.2 \mathrm{ks}$ under vacuum. 


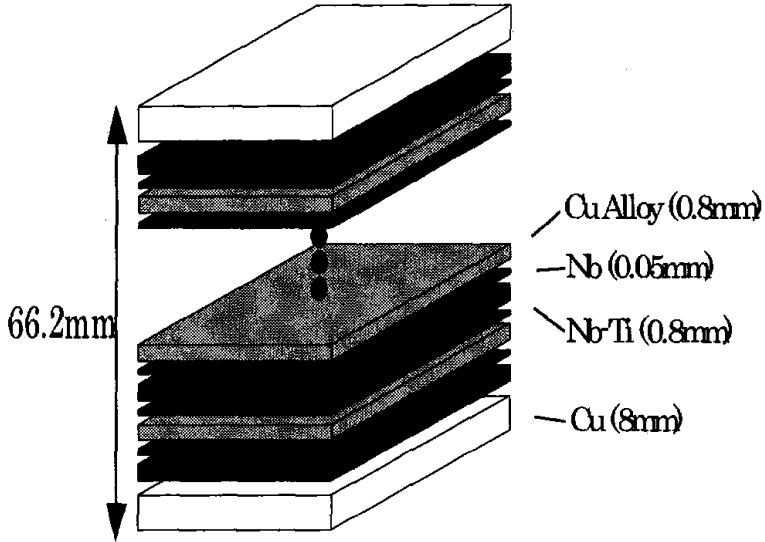

Fig. 1 Assembly of the starting stack of multilayer sheets

Finally the plate was cold-rolled to get thin sheets with thickness between 20 and $100 \mu \mathrm{m}$. Due to the present process, the final layer thickness of $\mathrm{Nb}-\mathrm{Ti}$ was designed from $120 \mathrm{~nm}$ to $4.8 \mathrm{~nm}$.

In order to assess the microstructure, TEM and SEM observations were carried out. The transport critical current measurements were carried out under external magnetic fields up to $8 \mathrm{~T}$ at liquid helium temperature. The critical current $\left(I_{c}\right)$ was determined with criterion of $1 \mu \mathrm{V} / \mathrm{cm}$ for the two type of samples where the current direction was parallel (L) or perpendicular $(C)$ to the rolling direction. The magnetic field was applied parallel to the tape surface and perpendicular to the current direction. The critical current density $\left(J_{\mathrm{c}}\right)$ was defined as $I_{d} / S$, where $S$ is the cross sectional area of the whole sample. The global pinning force $\left(F_{\mathrm{p}}\right)$ was calculated as $F_{\mathrm{p}}=$ $J_{\mathrm{c}} B$. The critical temperature $\left(T_{\mathrm{c}}\right.$ ) was measured by means of the inductive technique.

\section{EXPERIMENTAL RESULTS AND DISCUSSION}

Due to the present procedure, the reduction ratio of thickness reached maximumly $1.8 \times 10^{5}$. Fig. 2 shows the microphotograph of the cross section of the multilayer sheet, that was designed as the thickness of $\mathrm{Nb}-\mathrm{Ti}$ layer was $24 \mathrm{~nm}$. The well developed layered structure can be observed. From the similar photos, each layer thickness of the $\mathrm{Nb}-\mathrm{Ti}$ and $\mathrm{Cu}$ alloy was measured where the data of $\mathrm{Nb}-\mathrm{Ti}$ layer thickness are listed in TABLE I. The observed thickness was found to be nearly identical with the designed value with coefficient of variation less than 0.23 .

TABLE I

Designated and observed $\mathrm{Nb}$ - $\mathrm{Ti}$ layer thickness

\begin{tabular}{cccc}
\hline Sample Name & $\begin{array}{c}\text { Designated Layer } \\
\text { Thickness }(\mathrm{nm})\end{array}$ & $\begin{array}{c}\text { Observed NbTi Layer } \\
\text { Thickness }(\mathrm{nm})\end{array}$ & $\mathrm{COV}$ \\
\hline A1-4 & 40 & 43.3 & 0.21 \\
A $1-5$ & 20 & 19.7 & 0.23 \\
\hline
\end{tabular}

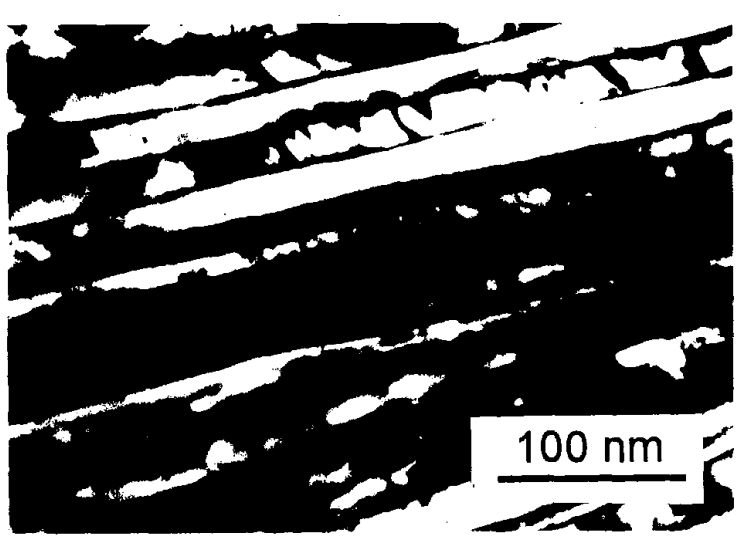

Fig. 2 Cross section of the multilayer sheet along to the rolling direction for the sample with $24 \mathrm{~nm} \mathrm{Nb}$-Ti layer thickness.

This indicates that the present thermomechanical treatment is suitable to manufacture the nanoscale multilayer.

Fig. 3 shows the change of critical temperature as a function of the designed layer thickness. With the $\mathrm{Nb}$ - $\mathrm{Ti}$ layer thickness was reduced from 120 to $30 \mathrm{~nm}$, the critical temperature gradually decreased. A rapid decrease was observed when the thickness became lower than $20 \mathrm{~nm}$, due to the proximity effect. The layer thickness dependence of critical temperature was evaluated for the present multilayer structure [6], where the change of order parameter was calculated based on the quantum mechanical theory and the critical temperature is estimated as a function of order parameter in the $\mathrm{Nb}$ - $\mathrm{Ti}$ layer. The calculated result shows that the critical temperature is constant with decreasing layer thickness up to $15 \mathrm{~nm}$ and then rapidly decreased to zero below $10 \mathrm{~nm}$. When comparing with the theoretical result, the decreasing rate was moderate, because the layer thickness distributes in practice and the proximity effect is averaged over the layers with different thickness.

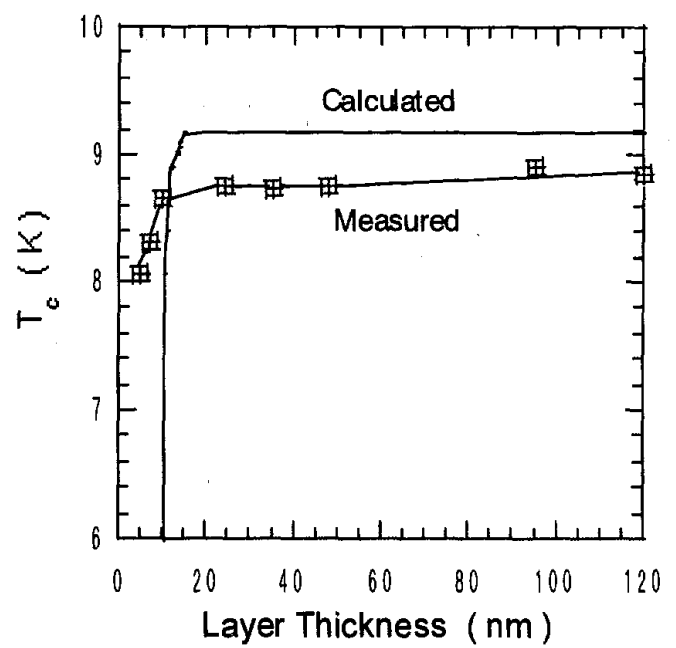

Fig. 3 Change of critical temeprature as a function of the designated layer thickness. 


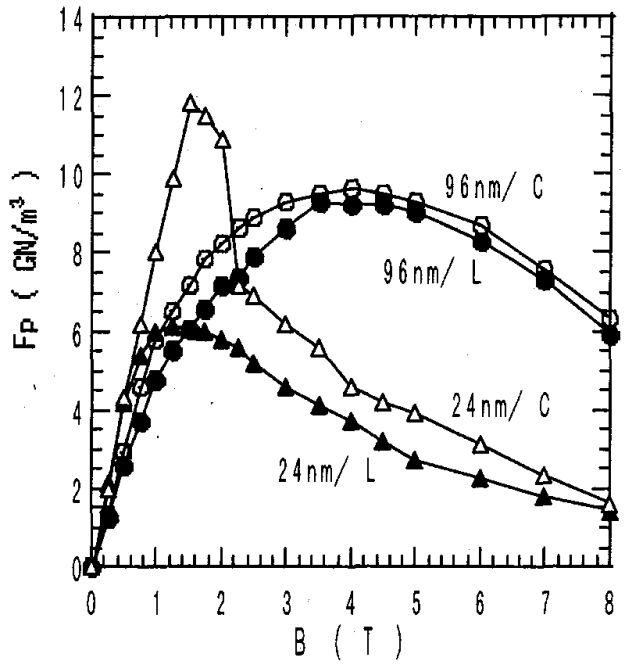

Fig. 4 Comparison of the direction of applied magnetic field for the global pinning force for the samples with different $\mathrm{Nb}-\mathrm{Ti}$ layer thickness, where $\mathrm{C}$ and $L$ indicate the magnetic fjeld perpendicular and parallel to the cold -rolling direction, respectively.

The global pinning force was compared for two samples with $\mathrm{C}$ and $\mathrm{L}$ directions taken from the same multilayer sheets as shown in Fig.4, where two cases with different $\mathrm{Nb}$-Ti layer thickness are shown. The samples with $96 \mathrm{~nm}$ thick showed the maximum nearly at $4 \mathrm{~T}$, while the global pinning force of the C's sample was always larger than that of the L's one. For the sample with $24 \mathrm{~nm}$ thick, the maximum appeared at the lower magnetic field less than $2 \mathrm{~T}$. The difference between two samples of $\mathrm{C}$ and $\mathrm{L}$ was more enhanced. Their result might be attributed to the directional dependency of work inhomogeneity, that is, the so-called sausaging takes place more frequently along the rolling direction. The critical current ( $I_{\mathrm{c}}=S J_{\mathrm{c}}$ ) is controlled at the minimum cross section when $J_{\mathrm{c}}$ is constant.

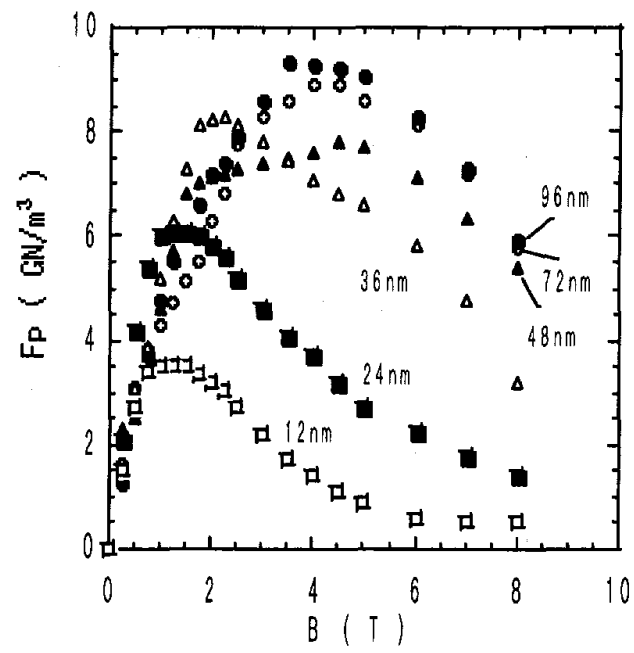

Fig. 5 Change of global pinning force for the L direction as a function of magnetic field for the annealed samples with different layer thickness.

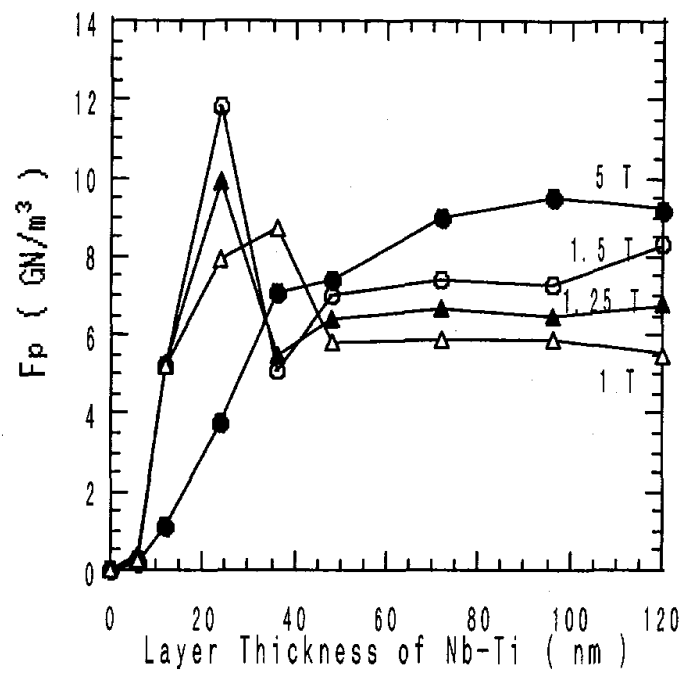

Fig. 6 Change of global pinning force as a function of layer thickness for the C's samples.

Change of global pinning force has been assessed as a function of $\mathrm{Nb}-\mathrm{Ti}$ layer thickness as shown in Fig. 5 for the annealed samples. When the layer thickness is larger than $70 \mathrm{~nm}$, the maximum appears at around $4 \mathrm{~T}$. On the other hand, the maximum position shifted toward the lower magnetic field and reached the magnetic field between 1 and $2 \mathrm{~T}$ for the samples with the thinner layer thickness. The same tendency has been observed for the behavior of global pinning force for the C's samples.

Fig. 6 shows the change of global pinning force as a function of layer thickness for the C's samples. At a constant magnetic field of $5 \mathrm{~T}$, the global pinning force decreased monotonically with decreasing layer thickness. For $1 \mathrm{~T}$, the global pinning force started to increase when the layer thickness decreased below $40 \mathrm{~nm}$. Then it reached a maximum at $35 \mathrm{~nm}$. For 1.5 $T$, the maximum value of $F_{p, \max }$ became highest of $12 \mathrm{GN} / \mathrm{m}^{3}$ for the specimen with $\mathrm{Nb}-\mathrm{Ti}$ layer thickness of $24 \mathrm{~nm}$. The global pinning force decreased largely to zero at the layer thickness less than $20 \mathrm{~nm}$.

The global pinning force due to the direct summation is proportional to the number density of pinning sites $\left(N_{\mathrm{p}}\right)$, the interaction volume $(V)$ and the square of thermodynamic critical field ( $B_{\mathrm{c}}$ ). The $B_{\mathrm{c}}$ is given as a function of the critical temperature. The layer thickness dependence of their quantities is schematically expressed as shown in Fig. 7. They have the respective characteristic thickness, where the layer thickness dependency changes. Here we consider their change at $1 \mathrm{~T}$. When the external magnetic field is applied parallel to multilayer interface, the layer thickness necessary to obtain the maximum pinning sites geometrically corresponds to $a_{\mathrm{f}} / 4$, where $a_{\mathrm{f}}$ is the lattice constant of the magnetic flux lattice. It is $49 \mathrm{~nm}$ for $1 \mathrm{~T}$. The interaction volume is constant at the large layer thickness, but it decreases when $d$ is smaller than $2 \xi$, 
where the coherence length $\xi$ is supposed to be $5.5 \mathrm{~nm}$ for $\mathrm{Nb}$-Ti alloys.

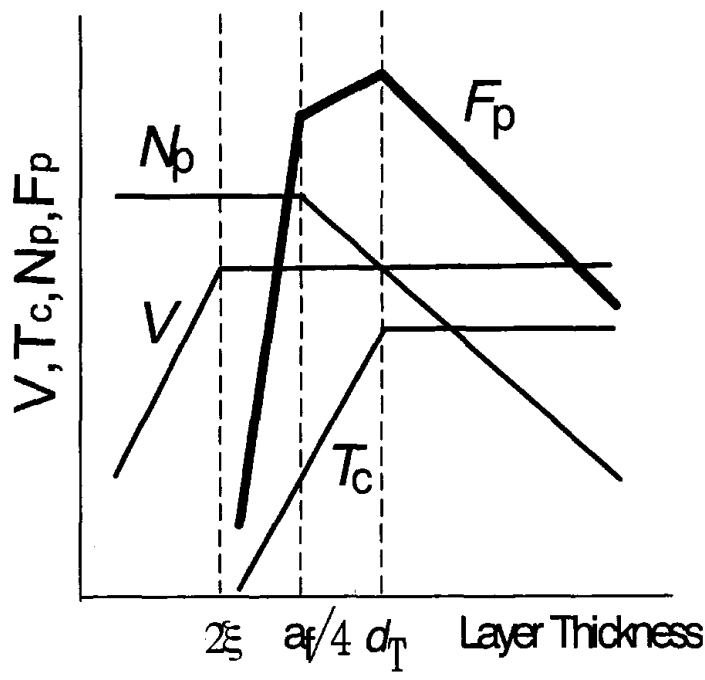

Fig. 7 Schematic representation on the layer thickness dependence of global pinning force at a constant magnetic fiel d.

As shown in Fig. 3, $T_{\mathrm{c}}$ is observed to decrease prominently at $d_{\mathrm{T}}$, that was about $20 \mathrm{~nm}$. Thus the layer thickness dependence of $F_{\mathrm{p}}$ can be qualitatively given as shown in Fig. 7. The maximum of $F_{\mathrm{p}}$ will appear at the layer thickness, at which the critical temperature starts to decrease. The decrease of $T_{c}$ depends on the proximity effect. When the proximity effect is reduced, the $d_{\mathrm{T}}$ should shift towards the thinner thickness and the improvement of global pinning force can be expected.

\section{CONCLUSION}

The $\mathrm{Nb}-\mathrm{Ti} / \mathrm{Nb} / \mathrm{Cu}$-alloy multilayers have been successfully fabricated by using the thermomechanical treatment technique.
In order to obtain homogeneous multilayers, the use of precipitation hardening type copper alloy was important. The thickness of Nb-Ti layer was reduced minimumly up to $4.8 \mathrm{~nm}$. The global pinning force increased monotonically with increasing layer thickness at $5 \mathrm{~T}$. On the other hand, it became highest at $24 \mathrm{~nm}$ when the magnetic field of $1.5 \mathrm{~T}$ was applied. The critical temperature decreased with decreasing layer thickness when it was smaller than $20 \mathrm{~nm}$. The present experimental results suggested that the maximum of global pinning force is given as a cross over of the increasing number density of pinning sites and the decreasing critical temperature due to proximity effect.

\section{ACKNOWLEDGMENT}

The authors express their thanks to Messrs. I Nakagawa and T.Unezaki for their help about the microstructure analysis.

\section{REFERENCES}

[1] H. Ohtsuka and I. Ito, "Critical current density and its microstructure dependence in $\mathrm{NbTi} / \mathrm{Nb} / \mathrm{Cu}$ superconducting multilayer sheets," Jap. Cryogenics Eng., vol. 32, pp.271-277, 1997.

[2] P.J.Lee and D. Larbalestier, "Determination of the flux pinning force of alpha- $\mathrm{Ti}$ ribbons in $\mathrm{Nb} 46.5 \mathrm{wt} \% \mathrm{Ti}$ produced by heat treatment of varying temperature, duration and frequency," J. Mater. Sci., vol.23, pp.3951-396, 1988

[3] L.R.Motowidlo et al, "Multifilament NbTi with artificial pinning centers: the effect of alloy and pin material on the superconducting properties," Appl. Phys. Lett., vol.61, 991, 1992

[4] K.Matsumoto et al, "Enhanced $J_{c}$ properties in superconducting NbTi composites by introducing $\mathrm{Nb}$ artificial pins with a layer structure," Appl. Phys. Lett., vol.64, pp.115-117, 1994.

[5] T. Kitai and K. Osamura, "Transition of flux pinning mechanism in mesoscopic $\mathrm{NbTi} / \mathrm{Nb} / \mathrm{Cu}$ superconductor," Cryogenics, vol 38 , pp.779-784, 1998.

[6] T. Kitai, PhD Thesis (Kyoto Univ.), 1998 